Cinémas

Revue d'études cinématographiques

Journal of Film Studies

\title{
André Bazin, What is Cinema?, traduit par Timothy Barnard, Montréal, Caboose, 2009
}

\section{Jacques Aumont}

Volume 20, numéro 1, automne 2009

Cinéma et oralité. Le bonimenteur et ses avatars

URI : https://id.erudit.org/iderudit/039280ar

DOI : https://doi.org/10.7202/039280ar

Aller au sommaire du numéro

Éditeur(s)

Cinémas

ISSN

1181-6945 (imprimé)

1705-6500 (numérique)

Découvrir la revue

Citer ce compte rendu

Aumont, J. (2009). Compte rendu de [André Bazin, What is Cinema ?, traduit par Timothy Barnard, Montréal, Caboose, 2009]. Cinémas, 20(1), 211-215.

https://doi.org/10.7202/039280ar d'utilisation que vous pouvez consulter en ligne.

https://apropos.erudit.org/fr/usagers/politique-dutilisation/ 
André Bazin, What is Cinema?, traduit par Timothy Barnard, Montréal, Caboose, 2009.

Qu'est-ce que le cinéma? La question, telle quelle, n'est plus d'actualité. En revanche, le recueil d'André Bazin, qui en avait fait son titre, est devenu un classique, lu et relu par des générations de cinéphiles et d'étudiants. Qu'est-ce que le cinéma?, dans ses diverses éditions et traductions, reste l'un des ouvrages les plus connus sur ce qu'on appelle encore le septième art, et d'ailleurs l'un des ouvrages qui a le plus contribué à établir l'idée qu'il s'agit bien d'un art.

En quoi une traduction anglaise, d'ailleurs partielle, de ce recueil est-elle digne d'être signalée? N'en existe-t-il pas déjà une, plus complète ${ }^{1}$ ? Faut-il vraiment revenir sur Bazin, si souvent commenté? Le faut-il en anglais, quand une édition française convenable (annotée, avec variantes et prenant la peine de vérifier le texte) n'existe toujours pas? Or, ce sont précisément ces trois raisons contre une nouvelle édition anglaise de Bazin qui ont déterminé Timothy Barnard à entreprendre la sienne.

Un lecteur français, en particulier, ne peut qu'être amèrement sensible à la troisième de ces clauses. Malgré les velléités manifestées ici ou là, malgré les déclarations grandiloquentes de tels érudits baziniens, on n'a toujours pas, cinquante ans après la mort du plus grand critique de cinéma français, une édition correcte de son ouvre. Pas d'édition complète, cela peut à la rigueur se concevoir; Bazin, qui multipliait les piges, reprenait souvent sans trop de scrupules des phrases, des paragraphes, quand ce n'était pas des textes presque tels quels, dans divers supports. Plus gênant est le désordre dans lequel ont été édités plusieurs recueils, établis selon des logiques différentes; le plus courant, celui que j'ai cité, n'existe même plus dans sa première version en quatre volumes, mais dans une version réduite qui a 
éliminé la moitié des textes. Surtout, toutes ces publications ont été faites sans souci éditorial: ni notes ni établissement des variantes, informations minimales sur la provenance, quasi aucune discussion des idées.

Sur les points techniques, l'édition de T. Barnard n'a aucun mal à être infiniment supérieure. À vrai dire, la comparaison est presque insultante pour lui, car il fait, justement, le travail qu'aucun des éditeurs français n'a jugé que Bazin méritait. Outils élémentaires, tels des index. Annotation précise et didactique du texte: tous les noms propres cités sont commentés - ce qui n'est pas inutile un demi-siècle après la parution des articles, certains contemporains de Bazin étant bien oubliés. Les sources des citations (rarement indiquées par l'auteur) sont, chaque fois que possible, identifiées. Les erreurs factuelles sont rectifiées, soit muettement dans le texte lorsqu'elles sont vénielles, soit dans des notes, toujours argumentées. Bref, on a fait le travail d'édition, au vrai sens de ce terme, et par là on témoigne qu'on tient ce texte pour digne de considération.

Au reste, l'établissement des sources et des références n'est pas qu'une affaire technique. Au début d'" Ontologie de l'image cinématographique", Bazin donne une citation de Malraux; après avoir recherché partout où l'on pouvait chercher, Barnard démontre que cette citation est, sous cette forme, inventée et, surtout, que Bazin a lu trop vite et n'a pas vu (ou pas voulu voir) que Malraux défend une idée du cinéma fondée sur le montage, donc bien différente de celle que lui-même prône. Plus remarquable encore est le travail de recherche mené à propos d'une citation de "Théâtre et cinéma». Bazin cite - en se trompant sur l'orthographe du nom de l'auteur et la date de l'article - un texte d'un M. Rozenkranz paru dans Esprit en 1934. Au terme d'une analyse serrée que je ne peux qu'évoquer (mais dont je puis dire qu'elle me semble cohérente), Barnard émet la passionnante et très vraisemblable hypothèse que Rozenkranz est un pseudonyme de Siegfried Kracauer, alors exilé en France. Comme il le dit modestement, si cette hypothèse se confirmait, ce serait une extraordinaire rencontre entre les deux grands tenants d'une théorie « réaliste» du cinéma, qui par ailleurs ne se sont jamais cités mutuellement. 
La deuxième raison qui a déterminé cette entreprise, c'est paradoxalement l'existence de la traduction de Hugh Gray. Sur ce point, je fais confiance à Timothy Barnard et à son appréciation négative de ladite traduction, que je n'ai pour ma part jamais fréquentée (je n'en connais que l'usage qu'en fait Dudley Andrew, un autre anglophone auquel la mémoire de Bazin doit beaucoup). J'ai une petite expérience de la traduction, avec Eisenstein, et je peux aisément sympathiser avec quelqu'un qu'une mauvaise traduction rend malheureux (si je le pouvais, je brûlerais tous les exemplaires de Le film, sa forme, son sens, sinistre travail d'amateur bourré d'erreurs en tout genre).

Sur la troisième raison, en revanche, on peut s'arrêter un peu plus longuement. Faut-il encore discuter Bazin? La glose ne lui a pas manqué: n'en a-t-on pas épuisé la substance? Ma question est rhétorique, bien sûr, mais elle s'appuie sur une évidence: Bazin est, encore aujourd'hui, très probablement le critique de cinéma de langue française sinon le plus lu, en tout cas le plus cité - même si, chez les plus jeunes gens, il est concurrencé par Daney et Deleuze. Pourquoi ? Évidemment pour quelques mauvaises raisons, dont l'habitude et l'inertie intellectuelle risquent de faire partie. Mais aussi pour une raison excellente, et même deux: les idées de Bazin sont importantes, et elles sont limpides. Importantes, parce qu'elles touchent à un point fondamental de l'histoire et de l'esthétique du cinéma, son supposé réalisme. Limpides, parce que personne, je crois, n’a écrit sur le cinéma des choses aussi profondes dans une langue aussi peu encombrée de jargon, et de façon aussi systématique. (Deleuze mène une entreprise avant tout philosophique, dans une langue peu amène et chargée en néologismes; Daney, dont la pensée est agile et le style, séduisant, n’a pas proposé un seul concept réutilisable ou généralisable.)

On me dira que je m'éloigne de mon objet, et que la tâche d'un traducteur n'est pas d'engager ou de poursuivre le débat intellectuel. D'abord, je ne partage pas ce point de vue réducteur (j'ai eu la prétention de commenter Eisenstein en le traduisant). Ensuite, il importe fort peu de savoir qui propose quoi; l'intéressant est la qualité de la discussion engagée. Timothy Barnard, qui n'a pas d'états d'âme à ce sujet, ne se prive pas de 
discuter, autant que de besoin, les idées qu'il rencontre dans le texte qu'il traduit. Une première et importante discussion - quoique relativement courte - vient à propos de «L'évolution du langage cinématographique». Rappelant qu'en français "montage" signifie deux choses très différentes l'opération technique de mise bout à bout des plans (en anglais, editing) et les principes sémantiques et formels mis en ouvre, sciemment ou non, dans cette opération (en anglais, montage) —, Barnard souligne, d'abord qu'une traduction doit sur ce point interpréter le texte et choisir entre ces deux sens, mais aussi et surtout, que "montage", dans le langage de Bazin, est un terme connoté négativement. C'est l'occasion de préciser cette opposition au montage, célèbre mais souvent mal comprise, d'en redéfinir les limites et aussi de l'apprécier historiquement.

Un peu plus loin, on trouve une réflexion sur la notion de découpage, plus fouillée (vingt pages, dont une et demie de références des textes théoriques consultés). Pour l'essentiel, elle provient de l'indignation de Barnard, constatant que certains de ses devanciers n’ont pas hésité à traduire découpage par... editing! Autrement dit, que certains traducteurs de Bazin n'ont littéralement rien compris à ce qu'ils traduisaient, puisqu'ils n'ont pas vu que tout le système théorique de Bazin repose sur une opposition fondamentale entre le découpage et le montage. Tous deux sont des opérations mentales permettant de donner au film son organisation; mais l'une intervient avant le tournage, et concerne principalement les grandes articulations du récit, quand l'autre se produit après le tournage et manifeste de manière plus forte, voire plus violente, le point de vue du cinéaste. Barnard parcourt à ce propos une vaste littérature critique et théorique, et ce véritable petit essai aide beaucoup à situer les idées de Bazin dans une histoire des grandes théories du cinéma.

On le voit, cette version anglaise de Bazin a certaines qualités qui la rendent utile aussi au lecteur francophone (on pourrait en tout cas rêver d'une édition française qui comprendrait ce formidable travail d'annotation). Cette édition n'est pour l'heure accessible, hélas, que dans les quelques pays qui n'ont pas 
cédé au chantage des grandes maisons d'édition anglo-saxonnes, et n'ont pas porté à soixante-dix ans ou davantage la durée du copyright - le Canada au premier chef.

Un mot pour finir - et pour regretter, platement, que ce volume ne soit pas plus épais. On peut imaginer les raisons qui ont mené à garder un volume raisonnable à ce livre, et Barnard n'a pas tort d'observer que son choix de textes est représentatif de toutes les grandes idées baziniennes. Je me contenterai donc de souhaiter qu'un second volume, aussi remarquablement édité, soit bientôt proposé à notre attention et à notre amour de Bazin.

Jacques Aumont

Université Sorbonne Nouvelle — Paris 3

NOTES

1. Bazin 1967 et 1971. Ces deux recueils contiennent vingt-six articles au total, pris respectivement dans les tomes I et II, et dans les tomes III et IV, de l'édition française en quatre volumes.

\section{RÉFÉRENCES BIBLIOGRAPHIQUES}

Bazin 1967 : André Bazin, What Is Cinema?, traduction de Hugh Gray, Berkeley, University of California Press, 1967.

Bazin 1971 : André Bazin, What Is Cinema? II, traduction de Hugh Gray, Berkeley, University of California Press, 1971. 